\title{
VIEW POINT
}

\section{GLOBAL ERADICATION OF YAWS: NEGLECTED DISEASE WITH RESEARCH PRIORITY}

\author{
Md. Robed Amin ${ }^{1}$, Ariful Basher ${ }^{2}$, Md Ferdous Zaman ${ }^{3}$, M A Faiz ${ }^{4}$
}

\begin{abstract}
:
Yaws is a disfiguring, debilitating non-venereal treponemal infection caused by Treponema pallidum subspecies pertenue. It is a contagious disease transmitted by direct (person-to-person) contact with the infectious yaws lesion. Early lesions of this disease manifest in the form of skin lesions, which on healing show little scarring. The disease can be progressive wherein bone and cartilage are affected leading to disability. The disease can be cured and prevented by a single injection of long acting (benzathine benzyl) penicillin. Between 1952 and 1964, a worldwide campaign led by WHO and the United Nations Children's Fund (UNICEF) to control and eventually eradicate yaws and other endemic treponematoses were undertaken.By the end of the 1970s, reemergence of yaws occured in many countries and it prompted a World Health Assembly Resolution requesting the implementation of integrated treponematoses control programmes. While the programme initially showed tremendous result but yaws control efforts paved the way for the development of the primary healthcare system in affected areas.

The disease is amenable to eradication epidemiologically, technologically, historically and from political point of view if appropriate research based programme in national and international arena is set.
\end{abstract}

Key words: Eradication, Yaws, Neglected,disease,Research

\section{Introduction:}

Yaws, caused by the spiral-shaped bacterium (spirochete) Treponema pallidum subspecies pertenue is a contagious, non-venereal infection in humans that presents mainly in children below 15 years primarily affecting skin, bones and cartilage. ${ }^{1}$ The disease occurs primarily in poor, rural and marginalized populations in parts of Africa, Asia and South America, where conditions of overcrowding, poor water supply, and lack of sanitation and hygiene prevail. If left untreated, yaws leads to crippling and disfiguring consequences. ${ }^{2}$ Skin to skin contact is the main route for transmission, together with breaks in the skin caused by injuries, bites, or excoriations . ${ }^{3}$ According to older accounts, flies may also play a role as vectors for transmission. ${ }^{4}$ Yaws, like syphilis, has been classified into the following 4 stages: $: 5,6,7,8$
1. Primary stage: The initial yaws lesion develops at the inoculation site.

2. Secondary stage: Widespread dissemination of treponemes results in multiple skin lesions similar to the primary yaws lesion.

3. Latent stage: Symptoms are usually absent, but skin lesions can relapse.

4. Tertiary stage: Bone, joint, and soft tissue deformities may occur.

Children of preschool and school going (under 15 years) are the main yaws victims, with peak incidence in the six- to ten-year-old age range. Treatment of earlystage yaws is very simple and inexpensive. Re-infection or relapse leading to recurrence is rare. An estimated 50-100 million persons were infected before mass

1. Assistant Professor, Department of Medicine, Dhaka Medical College

2. Postgraduate Student, FCPS(Tropical Medicine) 2nd part, BSMMU, Dhaka

3. Registrar, Department of Medicine, Dhaka Medical College, Dhaka

4. Professor, Department of Medicine, Sir Salimullah Medical College, Dhaka

Correspondence: Dr. Md. Robed Amin. Assistant Professor, Department of Medicine,Dhaka Medical College. E-mail: robedamin@yahoo.com 
treatment campaigns in the 1950s. In the 1970s, yaws cases declined to fewer than 2 million. In the 1980s, fewer than 500 cases per year were reported in the Western Hemisphere. A resurgence of yaws has occurred in West and Central Africa, Southeast Asia, and the Pacific Islands, with recent outbreaks in Thailand, India, Indonesia, Papua New Guinea, and the Solomon Islands. Sporadic cases are reported in South America. In 1995, WHO estimated the number of infectious cases to be 460000 worldwide of which, 400000 were in west and central Africa, 50000 in South-East Asia and the rest in other tropical regions. ${ }^{9}$

\section{Eradication: Early Attempts, Failure, and Lessons:}

Between 1952 and 1964, WHO and the United Nations Children's Fund (UNICEF) led a worldwide campaign to control and eventually eradicate yaws and other endemic treponematoses. ${ }^{10}$ This was a major disease control effort undertaken by WHO just after its establishment in $1948 .{ }^{11}$ Control programmes were established in 46 countries and, by the end of 1964 , the number of cases had been reduced from 50 million to 2.5 million (a $95 \%$ reduction). ${ }^{12}$ The yaws control efforts paved the way for the development of the primary health care system in affected areas. ${ }^{13} \mathrm{In}$ the late 1960s, there was a shift in strategy from the vertical programme to integration of yaws surveillance and control into primary health care to tackle the remaining $5 \%$ of cases. However, this approach did not succeed.By the end of the 1970s, reemergence of yaws in many countries prompted a World Health Assembly Resolution requesting the implementation of integrated treponematoses control programmes. ${ }^{14}$ Renewed control efforts were implemented in several countries, e.g. Benin, Burkina Faso, Côte d'Ivoire, Ghana, Mali, the Niger and Togo, but these efforts were not sustained. In 1984, a global meeting was organized in Washington, DC followed by regional meetings with the aim of reviving eradication activities. ${ }^{15}$ These attempts were half-hearted and the goal of eradication remained elusive. ${ }^{16}$ At this time, most of the yaws programmes had been integrated into primary health care which were generally too weak to implement the activities of a vertical programme. ${ }^{17}$ Since then, yaws has remained as a public health problem in a few pockets, including three countries in the SEA Region - India ,Indonesia and Timor-Leste. The South-East Asia Region of WHO kept yaws high on its agenda and set the goal of regional eradication by 2012 in its two remaining endemic countries - Indonesia and Timor-Leste after India achieved elimination.
The yaws eradication is possible as there are factors which is always amenable to take care of:

- Man is the principal reservoir

- Patients with primary and secondary lesions are the main sources of infection

- Progressive decline of reported cases over the years

- Direct contact with secretions of skin lesions is the main mode of transmission

- Intimate and prolonged contact needed for person to person spread

- Effective treatment for cure available and treatment is safe, effective and operationally feasible

- Few neglected foci do not cause epidemics and do not spread to other areas

- Mass campaigns aimed at total population examination to detect cases and treat them along with contacts have greatly reduced the prevalence and transmission

- Improvement in personal hygiene, sanitation, community awareness of the disease, especially free availability of effective treatment and improved socio economic conditions facilitate reduction of transmission

The main lessons learned from the past are that yaws can be eliminated with sustained efforts as shown in many countries and recently in India. Since 2004, India has reported no new cases. ${ }^{18}$ In the Western Pacific Region, three countries remain endemic Papua New Guinea, the Solomon Islands and Vanuatu.

\section{Issues and challenges:}

"Some of the issues and challenges that have hampered yaws eradication historically include the lack of an effective mechanism for surveillance and case detection, limited political commitment and resources, limited capacity of general health staff to recognize and treat yaws, ensuring drug supply and logistics management, creating community awareness through appropriate advocacy /IEC [Information, Education, and Communication] campaigns and extending the services to remote and hard to reach areas," a recent WHO report remarked. ${ }^{10}$ "Unfortunately, yaws remains among the most neglected diseases and there is little global attention or focus on this disease. The World Health Organization should publicize the currently known and unknown status of surveillance 
for this disease in each of the remaining suspected endemic countries, and encourage mapping and more detailed reporting of surveillance data" 19 In a consultation round-up held recently at the organization's headquarters in Geneva, basic agenda for future action was undertaken. ${ }^{20}$ The agenda includes a rapid assessment of the burden of disease and infection in selected countries; revival of activities to control yaws; and establishment of an elimination program for yaws, directed by WHO in the context of its larger Neglected Tropical Diseases initiative. Elimination of clinical cases (zero cases) supported by active case-finding and serological surveys has been declared as the goal, but no timeline has been set. Although it is generally agreed that political and financial inertia are the biggest obstacles to interrupting transmission of yaws, some eradication experts point out that biological and medical barriers also exist. For example, although it is usually claimed that humans are the only reservoir of infection, $17 \%$ of the members of a wild gorilla population in the Republic of Congo presented typical yaws skin lesions. ${ }^{21}$ Yaws may also affect African baboons, and widespread serological testing of wild populations has been performed; genetic analyses of a strain collected from a Guinean baboon shows that it is closely related to human yaws strains. ${ }^{22}$ If complete eradication is to be enforced, nonhuman reservoirs need to be cleared as well. Successful treatment campaigns have already been carried out on primates against various infectious diseases; in the case of yaws, affected baboons were darted and treated with penicillin and an additional antibiotic. ${ }^{23}$ In parallel, more information is needed on the way pathogens cross transmit between humans and primate populations, so that suitable strategies to prevent disease spreading and/or resurgence may be developed. As for humans, untreated victims may remain infective for months, favoring the spread of treponemes within populations. A vaccine is still lacking, and so is a reliable test able to distinguish yaws from the various other treponematoses and monitor its impact on populations, as discussed earlier. Furthermore, some researchers noted, the recurrent shortage of benzathine penicillin G in North America due to production problems could make mass treatment difficult. ${ }^{24}$

\section{Research priorities:}

We still do not fully understand the epidemiology of yaws in non-human primates, the relationship between transmission intensity, cross transmission between human and non human primates, disease pattern, and severity of the disease and subsequent complications. There is lack of rapid diagnostic test especially in early lesions. We still need to know more insights on the effect of mass treatment compared with targeted treatment, given the cost of the medication.

\section{Diagnostic dilemma and surveillance}

The lack of reliable serological or morphological tests to distinguish T. pallidum subspecies obviously limits diagnostic accuracy, which can have serious detrimental effects. For example, treatment for a pregnant woman with burntout yaws and one with active syphilis should be different, and assigning the wrong treatment may result in a negative outcome.

\section{Focus of research:}

- In-depth analysis of the T. pallidum genome, in the attempt to identify and decipher determinants of host specificity, pathogenicity, and virulence among the different subspecies

- Research on DNA polymorphisms may lead to serological tests that could be used in the field, facilitating disease control and eradication efforts by permitting the monitoring of its continued impact on populations

- Active and passive surveillance with integrity need to be addressed before eradication policy in process.

\section{Treatment of preschool children}

In Timor- Leste, the health care workers engaged in immunization campaigns have reported clinical yaws among $1 \%-2 \%$ of children below five, during $2003-$ 2006,There is increasing evidence that preschool children would benefit from treatment even though their burden of infection may not be as high as that in other age groups. Yaws infection can lead to impairment of vaccine development leading to persistent infection of same or other infectious disease.

\section{Focus of research:}

- Injection of penicillin of preschool children is painful and frightening; health systems research for effective delivery of alternating drugs in combination with other nutritional supplement through community-based organizations should be carried out.

- More studies are required to confirm the adverse effects of yaws on children $<5$ years of age and the 
safety of alternating drugs in children $<5$ years of age. Based on the findings of these research studies, strategies could be developed for yaws eradication children $<5$ years of age.

\section{Improving the interventions with penicillin among school-age children}

In yaws, the occurrence of disease is directly related to the intensity of infection and is highest in schoolage children. In young children, intimate contact is highest at school activity especially in poor hygienic children. Regular and intensified injection of penicillin using the school infrastructure and schoolteachers is one of the strategies. Some countries in the Region have shown a good reduction in infection rates following mass eradication programme through penicillin shots. The ongoing programmes needs to be sustainable, ensured and impact should be evaluated.

\section{Focus of research:}

- Impact assessment of eradication of yaws programmes on the prevention of reinfection.

- Identification of sustained, effective interventions through community mobilization and partnerships

- Operational research along with an intervention programme among non-enrolled schoolchildren. These children are risky, infectious, reservoirs and amenable to sustain infection in community.

\section{Improving the personal hygiene and environmental changes:}

In general, poor hygienic conditions favor the transmission of yaws through contact with skin contact and secretions from lesion. Bites, excoriation, injury is the easy method for quick transmission. Studies need to be carried out to identify the specific components of hygienic conditions associated with a higher risk of yaws.

\section{Focus of research:}

- Estimation of the frequency and improving cleanliness, personal hygiene and environmental sanitation among schoolchildren and the community.

- Motivating community efforts to reduce transmission through hygiene or reduction of other maneuver.

- Environmental control of flies with intervention of vector bionomics and control strategy needs to address.
- Review and assessment of the current coverage of SAFE strategy and suggestions for improving the strategy at community level.

\section{Improvement of drug safety}

Women of childbearing age are among the most atrisk groups. Although penicillin is safe in pregnancy, there is always fear to use injection penicillin in immuno-altered status, as there is chance of hypersensitivity. Alternating drugs are not also very safe like tetracycline and doxycycline.

\section{Focus of research:}

- Absolute safe status of Penicillin is to be ensued

- Erythromycin/azithromycin as alternative should also be evaluated for absolute safety with easy way of administration

- New alternating drugs need to be identified for easy and effective administration.

\section{Monitoring drug efficacy and early detection of drug resistance}

The rate of post-treatment reinfection and relapse need to be addressed and the efficacy of Penicillin should be monitored regularly as efficacy diminishes with frequent and periodic use. As a result, there are justifiable concerns about the possibility of emerging resistance, which is now common for Penicillin in other bacterial infection. Also its efficacy, safety, adversity, resistance in primates should also be addressed. Effective monitoring of large scale eradication programme need to be built.

\section{Focus of research:}

- Develop new drugs, and evaluate the efficacy and safety of available drugs in human and non-human primates.

- Identification of sensitive and resistance genes in populations is to be known. Sensitive molecular tools to monitor drug efficacy need to be developed.

- Strategy to develop vaccines.

- Clinical trials for the development of vaccines against yaws and collaboration between research and eradication programme should be priority.

Further assessment of the risk/benefits of elimination of yaws:

a. Primate status: The impact of yaws in primates needs to be further documented and stronger evidence should be published from large-scale studies. 
b. Development of Pre School Age Children(PSAC) needs to be confirmed and properly tested in different epidemiological settings.

c. Outreach/cost/feasibility Innovative ways of outreaching and delivering penicillin alone or with other medications-should be documented, with particular attention to feasibility and costeffectiveness. It is essential that such approaches are properly monitored and evaluated at country level, and their successes documented in order to enhance advocacy and guide international agencies and policy makers to promote appropriate tools for the control of yaws infections and other diseases in children.

d. Adverse Event Reporting Forms (AERS) need to be included in any campaign delivering penicillin drugs so as to routinely capture data on the number of children having problems with injection. In the meantime, WHO and UNICEF should continue advocating for the development of novel paediatric formulations (rapid dissolving tablets or alternating drug like macrolides).

e. Pharmacovigilance: Safety of giving penicillin with other drugs in population-based interventions needs to be evaluated in large-scale eradiation programmes by a review of observational evidence.

f. Monitoring drug efficacy: Drug efficacy of penicillin delivered in large-scale interventions and the early occurrence of treatment failures needs to be monitored; any suspect episode of drug resistance should be properly reported.

g. Monitoring coverage : An integrated monitoring system to collect and manage data related to the number of children treated needs to be developed to report on coverage of different age and high risk groups.

h. Evaluating penicillin with Vaccine interactions: Possible synergies and interactions related to delivering inj penicillin with different vaccines (measles, BCG, polio) in large-scale vaccination campaigns needs to be further evaluated and properly followed up in the mid and long term.

i. Malaria/TB/HIV: Interactions of yaws with the management of the three big killers malaria, TB, and HIV—needs further study and science-based evidence to advocate and suggest novel means of combating malaria, TB, and HIV infections in yaws endemic areas.

\section{Hopes for the future}

yaws can be effectively controlled, at least at the national level, and even swept out if sufficient

energy is applied. 25,26 "Yaws had been declared to be eliminated from India on Sept 19, 2006 as no case of the disease was reported since 2003 . We need to build the network and develop the necessary partnership for tackling yaws in a sustainable manner. Ultimately eradication of another disease - take for example that of smallpox in 1978-would by itself be an achievement for public health!. The other side of the coin is that a second failure against this vincible enemy could cast discredit and mistrust on other ongoing and future eradication efforts, directed against more pernicious and lessvulnerable pathogens. Let's just hope this is not the case.

\section{References}

1. Perine PL, Hopkins DR, Niemel PLA, St John RK, Causse G, Antal GM. Handbook of endemic treponematoses: Yaws, endemic syphilis and pinta. Geneva: WHO; 1984.

2. Antal GM, Lukehart SA, Meheus AZ. The endemic treponematoses. Microbes Infect 2002;4(1):83-94.

3. Walker SL, Hay RJ. Yaws: A review of the last 50 years. Int J Dermatol 2002; 39: 258-260.

4. Barnard CC. Yaws and flies: Past and present opinions on the role of flies in the transmission of framboesia tropica. J Trop Med Hyg 1952; 55: 100114.

5. Backhouse JL, Hudson BJ. Evaluation of immunoglobulin G enzyme immunoassay for serodiagnosis of yaws. J Clin Microbiol 1995; 33(7): 1875-8.

6. Bora D, Dhariwal AC, Lal S. Yaws and its eradication in India-a brief review. J Commun Dis 2005; 37(1):1-11.

7. Chulay JD. Treponema Species (Yaws, Pinta Bejel). In: Mandell GL, Bennett JE, Dolin R, eds. Mandell, Douglas, and Bennett's Principles and Practice of Infectious Diseases. Vol 2. $5^{\text {th }}$ ed. Philadelphia, Churchill Livingstone; 2000:2490-4.

8. Engelkens HJ, Judanarso J, Oranje AP, Vuzevski VD, Niemel PL, van der Sluis JJ, et al. Endemic treponematoses. Part I. Yaws. Int J Dermatol 1991;30(2):77-83.

9. Informal consultation on endemic treponematoses. Geneva: WHO (WHO/EMC/95.3).

10. Guthe T, Willcox RR. Treponematoses: a world problem. WHO Chron 1954;8:37-113. 
11. Four decades of achievement: 1948-1988. Highlights of the work of WHO.Geneva: WHO; 1988: 1-2.

12. Antal GM, Causse G. The control of endemic treponematoses. Rev Infect Dis1985;7 Suppl. 2;S220-6. PMID:4012161

13. Troupin JL, Reynolds FW, Guthe T. Yaws control an opportunity forpromoting rural health services. Bull World Health Organ 1953;8:35564.PMID:13042586

14. Control of endemic treponematoses. World Health Assembly Resolution WHA 31.58. Geneva: WHO; 1978.

15. Burke JP, Hopkins DR, Hume JC, Perine PL, St John R. Internationalsymposium on yaws and other endemic treponematoses. Rev Infect Dis1985;7 Suppl. 2;S217-351.

16. Meheus A, Antal GM. The endemic treponematoses: not yet eradicated. Wldhlth statist quart 1992;45:228-37.

17. Meheus A. Integration of yaws control and primary health care. Rev Infect Dis1985;7 Suppl. 2;S284-8.

18. Elimination of yaws in India. Wkly Epidemiol Rec 2008;83:125-32.

19. International Task Force for Disease Eradication (2007) Summary of the eleventh meeting of theITFDE (II). Available: http://www.cartercenter.org/ documents/itfde_summaryoct07.pdf. Accessed 1 August 2008.

20. World Health Organization (2007) Informal consultation on yaws. Available: http://www.who.int/ neglected_diseases/diseases/ic24_26_jan2007.pdf. Accessed 1 August 2008.

21. Levrero F, Gatti S, Gautier-Hion A, Menard N. Yaws disease in a wild gorilla population and its impact on the reproductive status of males.Am J Phys Anthropol 2007; 132: 568-575.

22. Harper KN, Ocampo PS, Steiner BM, et al.Onthe origin of the treponematoses: A phylogenetic approach. PLoS Negl Trop Dis2008; 2: e148.

23. Wallis J, Rick LD. Primate conservation:The prevention of disease transmission. Int J Primatol 1999; 20: 803-826.

24. Scolnik D, Aronson L, Lovinsky R, et al..Efficacy of a targeted, oralpenicillin-based yaws control program amongchildren living in rural South America. Clin Infect Dis 2003; 36: 1232-1238.

25. Bora D, Dhariwal AC, Lal S. Yaws and itseradication in India-A brief review. J CommunDis 2005; 37 : $1-11$.

26. Lahariya C, Pradhan SK. Can Southeast Asia eradicate yaws by 2010? Some lessons from the Yaws Eradication Programme of India. Natl Med J India 2007; 20: 81-86. 